\title{
Extracting the beat: An experience-dependent complex integration of multisensory information involving multiple levels of the nervous system
}

\author{
LAUREL J. TRAINOR ${ }^{[1]}$ \\ McMaster University \\ ANDREA UNRAU \\ McMaster University
}

\begin{abstract}
In a series of studies we have shown that movement (or vestibular stimulation) that is synchronized to every second or every third beat of a metrically ambiguous rhythm pattern biases people to perceive the meter as a march or as a waltz, respectively. Riggle (this volume) claims that we postulate an "innate", "specialized brain unit" for beat perception that is "directly" influenced by vestibular input. In fact, to the contrary, we argue that experience likely plays a large role in the development of rhythmic auditory-movement interactions, and that rhythmic processing in the brain is widely distributed and includes subcortical and cortical areas involved in sound processing and movement. Further, we argue that vestibular and auditory information are integrated at various subcortical and cortical levels along with input from other sensory modalities, and it is not clear which levels are most important for rhythm processing or, indeed, what a "direct" influence of vestibular input would mean. Finally, we argue that vestibular input to sound location mechanisms may be involved, but likely cannot explain the influence of vestibular input on the perception of auditory rhythm. This remains an empirical question for future research.
\end{abstract}

Submitted 2009 February 7; accepted 2009 February 20.

KEYWORDS: rhythm, meter, vestibular, experience-dependent, multisensory, sound location

IN his paper "A simpler explanation for vestibular influence on beat perception: No specialized unit needed", Riggle (this volume) misinterprets our papers (Phillips-Silver \& Trainor, 2005, 2007, 2008; Trainor, 2008; Trainor et al., 2009) on movement-auditory interactions, and then presents evidence that he claims contradicts our findings. However, nothing in his paper actually argues against any of our findings. Because questions related to the mechanisms of auditory-vestibular interaction - and their phylogenetic and ontogenetic origins - are of fundamental importance in understanding both auditory perception and musical behaviour, in this reply we will engage in a constructive discussion of the critical question concerning whether auditory-vestibular interactions for rhythm are explained by auditory-vestibular interactions for locating sounds in space. But first it is necessary to set the record straight with respect to our experimental findings and our interpretation of the results.

In the series of studies cited above, we have shown that when played a metrically ambiguous auditory rhythm (i.e., one with no physical accents), both adults and infants can be biased to perceive it as a march (with perceived accents every second beat) or as a waltz (with perceived accents every third beat) by engaging them in rhythmic movement synchronized to every second or every third beat of the ambiguous auditory rhythm, respectively. Furthermore, we have shown that stimulation of the vestibular nerve in such a way as to give rise to the sensation of head movement synchronized to every second or to every third beat of the metrically ambiguous rhythm (in the absence of actual movement) also biases metrical interpretation for a march or a waltz. We therefore conclude that rhythmic movement can influence auditory rhythm perception, and that the vestibular system appears to play a critical role in this interaction.

Riggle's article is centered on the claim that we postulate a "specialized brain unit" for "beat perception" that is "directly" influenced by vestibular input. The first point to note is that our experiments do not simply concern "beat perception" but rather hierarchical metrical perception. And, 
contrary to Riggle's claims, we do not discuss a "specialized brain unit" for "beat perception"; indeed, we state that the physiological locus of the interaction between auditory and movement rhythms remains unknown, and that auditory and vestibular information converges at various subcortical and cortical areas (e.g., see Phillips-Silver \& Trainor, 2008; Trainor et al., 2009). In fact, we believe that rhythm perception likely involves a fairly distributed process in the brain. Models of rhythmic motor production involve connections between a number of brain areas including the cerebellum, supplementary motor area and premotor cortex (e.g., see Zatorre, Chen, \& Penhune, 2007). And fMRI studies clearly show that the presentation of auditory rhythms activates dorsal prefrontal cortex, cerebellum and basal ganglia in addition to auditory areas (e.g., Chen, Zatorre, \& Penhune, 2006, Grahn \& Brett, 2009; Lahav, Saltzman, \& Schlaug, 2007; Zatorre et al., 2007). Furthermore, our MEG studies show that the presentation of isochronous auditory beats elicits oscillatory patterns in the beta and gamma bands that reflect increases and decreases in neural synchronization between regions (Fujioka, Trainor, Large, \& Ross, in press).

As for Riggle's statement that we claim a "direct" connection between vestibular and auditory input in rhythm perception, we are not even sure what a "direct" connection would mean for a complex task such as extracting the metrical structure of a rhythm pattern. Indeed, vestibular and auditory inputs interact at a number of levels, both subcortically (e.g., dorsal cochlear nucleus; Oertel and Young, 2004) and cortically (see Phillips-Silver \& Trainor, 2007; Trainor et al., 2009 for discussions), and other sensory systems such as vision also converge with auditory and vestibular inputs. (Perhaps Riggle misinterpreted our description of applying a small current behind the ears as "direct stimulation of the vestibular nerve" to mean that we thought we were "directly" stimulating a "specialized brain unit" for auditory rhythm perception?) In our 2008 paper, we state "Exactly where auditory and vestibular information is integrated in the nervous system remains a mystery, although many projections from brainstem to cortex have polymodal components (Link \& Schwegler, 2000)". At this point, the only levels that can be clearly ruled out as contributing to the interaction are the inner ear and auditory and vestibular nerves because interactions occur here only if very high sound intensities are involved (Todd \& Cody, 2000). What our experiments clearly show is that movement involving vestibular stimulation influences the interpretation of an auditory rhythm that is metrically ambiguous, that is, an auditory rhythm that could be interpreted as a march or as a waltz, depending on whether every second or every third beat is perceived to be accented.

Riggle also claims that we postulate an "innate direct mechanism". However, nowhere do we claim that the interaction between auditory and vestibular systems in processing musical rhythm is innate. In fact, we suggest that experience likely plays a large role in the development of the neural connections that support auditory-vestibular interactions, as infants experience concurrent movement and sound from the onset of hearing. For example, caregivers rock infants while they sing or talk to them, and infants are typically carried for substantial periods of time every day, during which they experience correlated auditory (e.g., sound of footsteps) and vestibular (e.g., movement of the walking) input. Furthermore, a recent study indicates that the degree of influence of movement on the interpretation of ambiguous auditory rhythms in infancy is affected by specific experiences in Kindermusik classes (Gerry, Faux \& Trainor, 2008). At this point, the relative effects of innate and experiential influence on auditory-movement interactions remain largely unknown.

In sum, Riggle's statement that, if beat perception originates from body movement, "then [this] implies the existence of a specialized function in the brain that tracks musical beats and is directly influenced by vestibular system input rhythms" does not make sense. Interactions between the auditory and vestibular systems in metrical perception in no way imply the existences of an "innate" specialized "brain unit" that is "directly" influenced by vestibular input.

Now to the interesting part of Riggle's paper. He discusses research by Graybiel (Clark \& Graybiel, 1949; Graybiel \& Niven, 1950), Lewald and Karnath (2001), and Suzuki (Suzuki et al., 2008) showing that in determining where sounds are coming from in space, the auditory system is influenced by vestibular input, just as the visual system is influenced by vestibular input when calculating where objects are located in space. Indeed, it is critical to use vestibular information for auditory space perception. For example, consider the case of a stationary sounding object. If one moves one's head, the localization cues (particularly interaural time and intensity cues) change even though the object has not moved. So in order to correctly locate the object in space, it is necessary to use vestibular input to take out the effect of one's own head movement. This is nicely demonstrated by the studies cited by Riggle (and at the beginning of this paragraph) showing that perceived movement of one's body affects where sounds are perceived to originate. These studies find localization "illusions" when the system is tricked in its perception of movement, thereby demonstrating that vestibular information normally corrects sound localization for head movements. Indeed, Lewald and Karnath (2001) summarize their findings in their abstract by stating "...that vestibular information is taken into account by the brain for accurate 
localization of stationary sound sources during natural head and body motion". Suzuki et al. (2008) show that spatial perception of objects involves interaction between auditory, visual and vestibular systems, and that visual information dominates over auditory information. This finding is not surprising since the visual system can more accurately locate objects in space than can the auditory system.

Riggle suggests that the results of our 2009 paper (Trainor et al., 2009) can be explained by an auditory-vestibular illusion for the sound location. We will first discuss why we think that this is unlikely, although it is possible that this effect is involved. We will then discuss why even the involvement of auditory-vestibular interactions for sound location would in no way undermine our conclusions that movement in general, and vestibular input in particular, influences auditory rhythm perception.

Riggle suggests that when the vestibular nerve is stimulated in the absence of physical movement, as in our 2009 paper, it could give rise to a change in the perceived location of a sound. If so, subjects could perceive different beats as coming from slightly different spatial locations and this could bias which beats they hear as accented, and hence whether they perceive the ambiguous rhythm as a march or as a waltz. We think that this explanation is unlikely and, furthermore, even if true, it does not contradict our interpretation of our results. First, the location changes in question are not large, and spatial location is a rather weak cue for determining which beats are accented in music, the main auditory cues being duration, intensity and pitch (e.g., see Lerdahl \& Jackendoff, 1983). Second, in our 2007 paper (Phillips-Silver \& Trainor, 2007) we presented adults with the ambiguous auditory rhythm in the free field, and in our 2005 and 2008 papers (Phillips-Silver \& Trainor, 2005, 2008) we presented adults with the ambiguous auditory rhythm over headphones. In the case of the free field, no location illusion would be expected because the vestibular input would be congruent with subjects' self-induced bouncing movement, and the vestibular input would correct the changing cues to auditory location as they moved through space. In the case of headphones, there would be a mismatch between vestibular input as they moved and the auditory stimulus, which did not change as they moved because the sound source at the headphones moved with them. However, the influence of movement on whether the ambiguous auditory rhythm was perceived as a march or as a waltz did not differ significantly between free field and headphone conditions. Furthermore, whether or not participants were blindfolded also made no significant difference (Phillips-Silver \& Trainor, 2005, 2007), suggesting that conflicting visual information to location does not matter greatly. Therefore, disambiguation of an ambiguous auditory rhythm does not appear to depend on whether or not a small sound location illusion might be present.

In the experiment involving stimulation of the vestibular nerve (Trainor et al., 2009), no subjects reported hearing a location shift when we asked them what they heard. But even if a small location illusion was present and had an influence on the results, this in no way negates our conclusion that vestibular input influences the auditory perception of a rhythm. In fact, it strengthens it, and suggests that the interaction between musical and movement rhythms is based on sound location mechanisms, which are presumably evolutionarily ancient. Unfortunately, we do not know where the critical interaction between systems takes place for musical rhythm, or indeed if there is more than one critical area. Riggle seems to suggest that auditory-vestibular interactions for location information are based on low-level mechanisms. However, space perception is rather complicated, and although some cues to object location, such as interaural time difference, are known to be processed in subcortical structures, the cortex is needed for space perception, as is evidenced in patients with cortical lesions (e.g., Clarke et al., 2002).

Although sound location mechanisms might be an attractive basis for the interaction between vestibular and auditory rhythms, one further piece of evidence argues against it. Mammals in general are of course good at locating objects in space using vision and hearing in interaction with vestibular input to deal with effects of body movement on location cues. Although we know of no such studies, it would be very surprising if these animals did not show the location illusions that people show when tricked about their perceived self-movement, as integration of vestibular and auditory information is necessary for locating sounds in space. Yet very few species are capable of entraining rhythmic movement to an externally provided auditory beat. Indeed, it appears that this ability is only present in vocal learners such as cockatoos, seals and elephants (Schachner, Brady, Pepperberg, \& Hauser, 2008), suggesting that flexible rhythmic entrainment may require mechanisms for vocal imitation. Thus, although vestibular-auditory interactions for sound location might play a role in the influence of rhythmic movement on perceived auditory rhythm, it is unlikely to be the whole story.

In summary, we do not postulate a "specialized brain unit" for rhythm that is "directly" influenced by vestibular input. Rather, our data are consistent with the claim that movement in general, and vestibular stimulation in particular, affect the interpretation of metrically ambiguous auditory rhythms in humans. Whether or not auditory-vestibular mechanisms for sound location play a role in 
this interaction is an empirical question for future research.

\section{ACKNOWLEDGMENTS}

Writing this paper was supported by the Natural Sciences and Engineering Research Council of Canada.

\section{NOTE}

[1] Please address all correspondence to Laurel J. Trainor, McMaster University, Hamilton, ON. Canada L8S 4B2. Email: LJT@mcmaster.ca

\section{REFERENCES}

Chen, J. L., Zatorre, R. J., \& Penhune, V. B. (2006). Interactions between auditory and dorsal premotor cortex during synchronization to musical rhythms. Neuroimage, Vol. 32, pp. 1771-1781.

Clarke, S., Bellmann, T. A., Maeder, P., Adriani, M., Vernet, O., Regli, L. et al. (2002). What and where in human audition: selective deficits following focal hemispheric lesions. Experimental Brain Research, Vol. 147, pp. 8-15.

Clark, B., \& Graybiel, A. (1949). The effect of angular acceleration on sound localization; the audiogyral illusion. Journal of Psychology, Vol. 28, pp. 235-44.

Fujioka, T., Trainor, L.J., Large, E., \& Ross, B. (in press). Beta and gamma rhythms in human auditory cortex during musical beat processing. In Neuromusic III. Annals of the New York Academy of Sciences.

Gerry, D. W., Faux, A. L., \& Trainor, L. J. (June, 2008). Effects of Kindermusik Training on Rhythm and Movement Interactions in Infancy. Presented at The Neurosciences and Music III, Montreal, Canada.

Grahn, J. A., \& Brett M. (2009). Impairment of beat-based rhythm discrimination in Parkinson's disease. Cortex, Vol. 45, pp. 54-61.

Graybiel, A., \& Niven, J. (1951). The effect of a change in direction of resultant force on sound localization: the audiogravic illusion. Journal of Experimental Psychology, Vol. 42, pp. 227-30.

Lahav, A., Saltzman, E., \& Schlaug, G. (2007). Action representation of sound: Audiomotor recognition network while listening to newly acquired actions. Journal of Neuroscience, Vol. 27, pp. 308-314.

Lerdahl, F., \& Jackendoff, R. (1983). A Generative Theory of Tonal Music. Cambridge, MA: MIT Press.

Lewald, J., \& Karnath, H. O. (2001). Sound lateralization during passive whole-body rotation. European Journal of Neuroscience, Vol. 13, pp. 2268-2272.

Oertel, D., \&Young. E. D. (2004). What's a cerebellar circuit doing in the auditory system? Trends in Neuroscience, Vol. 27, 104-110.

Phillips-Silver, J., \& Trainor, L. J. (2008). Vestibular influence on auditory metrical interpretation. Brain and Cognition, Vol. 67, pp. 94-102.

Phillips-Silver, J., \& Trainor, L. J. (2007). Hearing what the body feels: Auditory encoding of rhythmic movement. Cognition, Vol. 105, pp. 533-54.

Phillips-Silver, J., \& Trainor, L. J. (2005). Feeling the beat in music: Movement influences rhythm per- 
ception in infants. Science, Vol. 308, pp. 1430.

Schachner, A., Brady, T. F., Pepperberg, I., \& Hauser, M. (2008). Spontaneous entrainment to auditory rhythms in vocal-learning bird species. Presented at The Neurosciences and Music III, Montreal, Canada.

Suzuki, M., Inoue, R., Kashio, A., Saito, Y., Nakanishi, W., Yamada, C., et al. (2008). Combined effects of vestibular stimulation and gaze direction on orientation of sound lateralization. Neuroscience Letters, Vol. 436, pp. 158-162.

Todd, N. P. M. \& Cody, F. W. (2000). Vestibular responses to loud dance music: A physiological basis of the "rock and roll threshold"? Journal of the Acoustical Society of America, Vol. 107, pp. 496-500.

Trainor, L. J. (2007). Do preferred beat rate and entrainment to the beat have a common origin in movement? Empirical Musicology Review, Vol. 2, pp. 17-20.

Trainor, L. J., Gao, X., Lei, J., Lehtovarara, K., \& Harris, L. R. (2009). The primal role of the vestibular system in determining musical rhythm. Cortex, Vol. 45, pp. 35-43.

Zatorre, R. J., Chen, J. L., \& Penhune, V. B. (2007). When the brain plays music: Auditory-motor interactions in music perception and production. Nature Reviews Neuroscience, Vol. 8, pp. 547-558. 